\title{
EFFECT OF THERMAL RADIATION, JOULE HEATING, HEAT SOURCES ON HYDROMAGNETIC FLOW OF MICROPOLAR FLUID PAST A STRETCHING SURFACE WITH CONVECTIVE BOUNDARY CONDITION
}

$$
\begin{gathered}
\text { P. Somasekhara Reddy }{ }^{\# 1} \text {, Prof. D.R.V.Prasada Rao(Rtdl) }{ }^{\# 2} \\
{ }^{\# 1} \text { Research Scholar, Department of Mathematics, Rayalaseema University, Kurnool, A.P. } \\
{ }^{\# 2} \text { Department of Mathematics, S.K.University, Anantapuramu - 515 003,A.P,India. }
\end{gathered}
$$

\section{ABSTRACT:}

In this paper, an attempt has been made to investigate the influence of thermal radiation, dissipation, heat sources and convective boundary condition on the flow of a micropolar fluid past a stretching sheet. The non-linear coupled equations have been solved numerically. The effect of various parameters on the flow characteristics are depicted through graphs and tables.

Keywords: Joule Heating, Heat Source, Micropolar Fluid, Stretching Sheet, Dissipation

Corresponding Authour:P. Somasekhara Reddy, Email:p.somasekhara.reddy@gmail.com

\section{INTRODUCTION:}

Free convection flows are great interest because of their various engineering, scientific and industrial applications in heat and mass transfer. Free convection heat and mass transfer occurs in the fields of design of chemical processing equipment, formation and dispersion of fog, distributions of temperature and moisture over agricultural fields, groves of fruits .Free convection heat and mass transfer fluids have been undertaken by several authors.It is well known that most fluids which are encountered in chemical and allied processing applications do not satisfy classical Newton's law and are accordingly known as non-Newtonian fluids. The study of non-Newtonian fluid flows has gained much attention from the researchers because of its applications in biology, physiology, technology and industry. In addition, the effects of heat and mass transfer in non-Newtonian fluid also have great importance in engineering applications, for instance, the thermal design of industrial equipment dealing with molten plastics, polymeric liquids, food stuffs, or slurries. A number of mathematical models have been proposed to explain the rheological behaviour of nonNewtonian fluids. The fluid model introduced by Eringen (8) exhibits some microscopic effects arising from the local structure and macro motion of the fluid elements. Further, they sustain coupled stresses and include classical Newtonian fluid as a special case. The model of micropolar fluid represents fluids consisting of rigid, randomly oriented (or spherical) particles suspended in a viscous medium where the deformation of particles is polymeric additives, geomorphological sediments, colloidal suspension, haematological suspensions, liquid crystals, lubricants etc. The mathematical theory of equations of porous media are presented by Lukaszewicz(11). 
In industrial and chemical engineering processes in which heat and mass transfer is a consequence of buoyancy effects caused by diffusion and concentrations vary from point to point resulting in mass transfer. The Soret and Dufour effects are very significant when the temperature and concentration gradients are high. Thus diffusion-thermo or Dufour effect corresponds to the energy flux cause by a concentration gradient in a binary fluid or mixture where as thermal-diffuson or Soret effect corresponds to species differentiation developing in an initial homogeneous mixture submitted to a thermal gradient. This aspects has been analysed by many researchers $(1-7,9,10,12-18)$.

In this paper we investigate the effect of partial slip, thermal radiation, dissipation, heat sources and convective boundary condition on the flow of a micropolar fluid past a stretching sheet. The non-linear governing equations have been analysed by Finite element method with three nodded line segments.

\section{FORMULATION OF THE PROBLEM:}

We analyse an incompressible two-dimensional MHD micropolar's fluid flow over a permeable stretching surface with slip velocity.The entire fluid system is under the influence of a uniform magnetic field. Thermal radiation and joule heating effects due to magnetic and electric fields are also encountered (see fig.I).

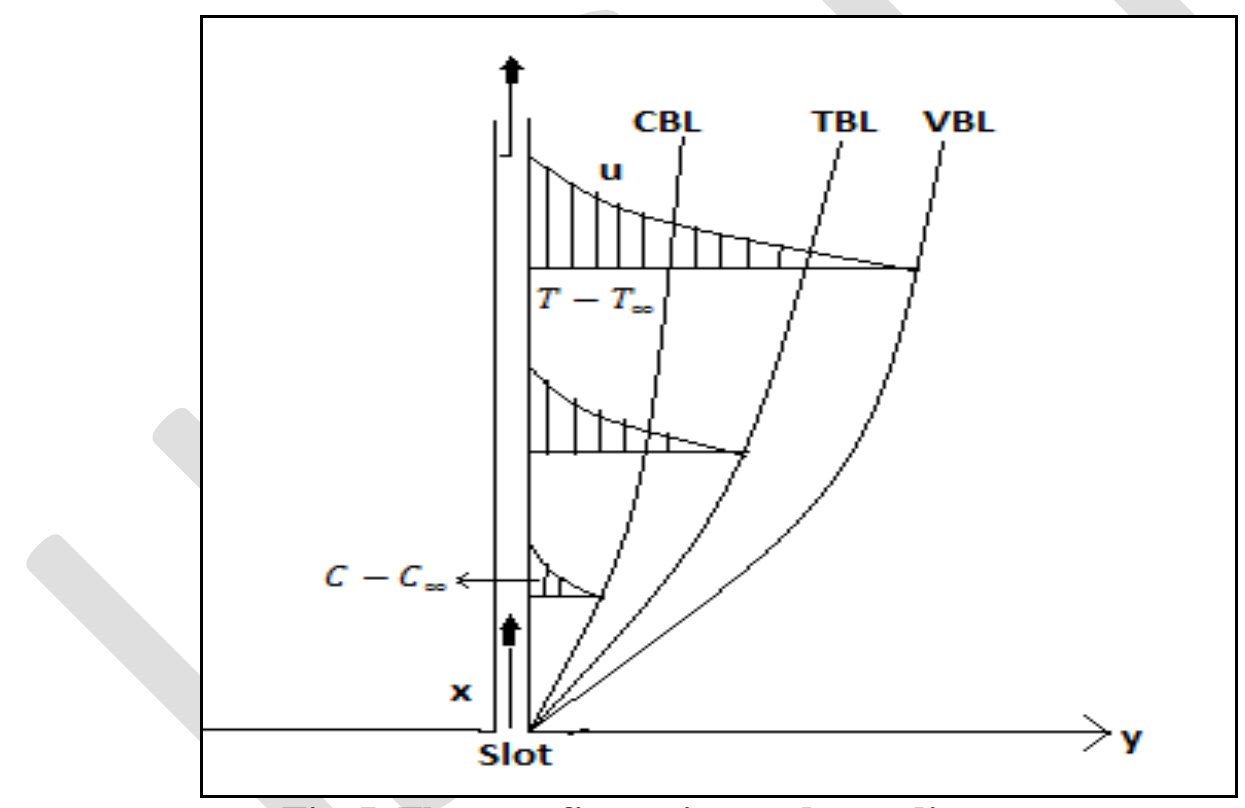

Fig. I .Flow configuration and coordinate system.

The equations governing the flow, heat and mass transfer under Boussinesque approximation and boundary layer approximations are

$$
\begin{gathered}
\frac{\partial u}{\partial x}+\frac{\partial v}{\partial y}=0 \\
u \frac{\partial u}{\partial x}+v \frac{\partial u}{\partial y}=\left(\frac{\mu+k}{\rho}\right) \frac{\partial^{2} u}{\partial y^{2}}+\frac{k}{\rho} \frac{\partial \omega}{\partial y}+\frac{\sigma}{\rho}\left(E_{0} B_{0}-B_{0}^{2} u\right)+G(\theta+N \phi) \\
u \frac{\partial \omega}{\partial x}+v \frac{\partial \omega}{\partial y}=\left(\frac{\gamma}{\rho j}\right) \frac{\partial^{2} \omega}{\partial y^{2}}-\frac{k}{\rho j}\left(2 \omega+\frac{\partial u}{\partial y}\right)
\end{gathered}
$$




$$
\begin{aligned}
& u \frac{\partial T}{\partial x}+v \frac{\partial T}{\partial y}=\left(\frac{k_{f}}{\rho C_{p}}\right) \frac{\partial^{2} T}{\partial y^{2}}-\left(\frac{Q^{\prime}}{\rho C_{p}}\right)\left(T-T_{\infty}\right)+\left(\frac{\left.u B_{o}-E_{0}\right)^{2} \sigma}{\rho C_{p}}+\left(\frac{16 \sigma^{\circ} T_{\infty}^{3}}{3 \beta_{R}}\right) \frac{\partial^{2} T}{\partial y^{2}}\right. \\
& u \frac{\partial C}{\partial x}+v \frac{\partial C}{\partial y}=\left(D_{B}\right) \frac{\partial^{2} C}{\partial y^{2}}-k_{c}\left(C-C_{\infty}\right)
\end{aligned}
$$

The boundary conditions are

$$
\begin{array}{lr}
u=a x+\alpha\left((\mu+k) \frac{\partial u}{\partial y}+k \omega\right), v=-v_{w}, \omega=-s \frac{\partial u}{\partial y}, \\
-k_{f} \frac{\partial T}{\partial y}=h_{f}\left(T_{f}-T\right), C=C_{w} & \text { at } y=0 \\
u \rightarrow 0, \omega \rightarrow 0, T \rightarrow T_{\infty}, C \rightarrow C_{\infty} & \text { as } y \rightarrow \infty
\end{array}
$$

Where $\mathrm{u}$ and $\mathrm{v}$ are the velocity components along $\mathrm{x}$ and $\mathrm{y}$ directions, $\mu, \omega, k, \rho, j, \gamma, \sigma, B o, E o, k_{f}, C_{p}, \sigma^{\bullet}, \beta_{R}, v_{w}, h_{f}, Q^{\prime}, T, T_{f}$, $C_{w}, C_{\infty}$ and $T_{\infty}$ are the viscosity, micro-rotation or angular velocity, vortex viscosity, density, microinertia density, spin gradient viscosity, electrical conductivity, applied magnetic field strength, applied electric field, thermal conductivity, specific heat at constant pressure, slip coefficient, suction /injection velocity, Stefan-Boltzmann constant, mean absorption coefficient, heat transfer coefficient, strength of the heat source, fluid and ambient temperature respectively, wall concentration, fluid and ambient concentration respectively. A linear relationship between the micro-rotation function $\omega$ and the surface stress $\left(\frac{\partial u}{\partial y}\right)$ is chosen for investigating the effect to different surface conditions for the microrotation. Here $\mathrm{S}$ is the boundary parameter and varies from 0 to 1 . The first boundary condition $(S=0)$ is a generalisation of the no slip condition, which requires that the fluid particles close to a solid boundary stick to it neither translating nor rotating. The second boundary condition i.e., micro-rotation is equal to the fluid vortices at the boundary $(S \neq 0)$ means that in the neighbourhood of the boundary, the only rotation is due to fluid shear and therefore, the gyration vector must be equal to fluid vortices.

On setting

$$
\eta=y \sqrt{\frac{a}{v}}, \psi=\sqrt{a v} x f(\eta), \omega=a x \sqrt{\frac{a}{v}} h(\eta), \quad \theta=\frac{T-T_{\infty}}{T_{w}-T_{\infty}}, \phi=\frac{C-C_{\infty}}{C_{w}-C_{\infty}}
$$

Equation (1) is automatically satisfied and equations(2)-(5) take the form

$$
\begin{aligned}
& (1+\Delta) f^{\prime \prime \prime}+0.5\left(f f^{\prime \prime}-\left(f^{\prime}\right)^{2}\right)+G(\theta+N \phi)+\Delta h^{\prime}+M^{2} E-M^{2} f^{\prime}=0 \\
& 0.5\left(f f-f h^{\prime}\right)=\lambda h^{\prime \prime}-\frac{\lambda}{G_{1}}\left(2 h+f^{\prime \prime}\right) \\
& \left(1+\frac{4 R d}{3}\right) \theta^{\prime \prime}+0.5 \operatorname{Pr} f \theta^{\prime}-Q \operatorname{Pr} \theta+M^{2} E c \operatorname{Pr}\left(\left(f^{\prime}\right)^{2}+E^{2}-2 E f^{\prime}\right)=0 \\
& \phi^{\prime \prime}+0.5 S c f \phi^{\prime}-\gamma S c \phi+S c S o \theta^{\prime \prime}=0 \\
& f=f_{w}, f^{\prime}(0)=1+\alpha(1+\Delta) f^{\prime \prime}(0), h(0)=-s f^{\prime \prime}(0), \theta^{\prime}(0)=-B i(1-\theta(0)), \phi(0)=1 \\
& f^{\prime}(\infty)=0, h(\infty)=0, \theta(\infty)=0, \phi(\infty)=0
\end{aligned}
$$

where Where $G=\frac{\beta g\left(T_{w}-T_{\infty}\right)}{a^{2} x}, M^{2}=\frac{\sigma B_{o}^{2}}{\rho a}, N=\frac{\beta^{*}\left(C_{w}-C_{\infty}\right)}{\beta\left(T_{w}-T_{\infty}\right)}, \Delta=\frac{k}{\mu}$ 


$$
\begin{aligned}
& G 1=\frac{\gamma}{v k}, \lambda=\frac{\gamma}{\rho j v}, E^{2}=\frac{E_{0}^{2}}{u_{w}^{2} B_{o}^{2}}, \operatorname{Pr}=\frac{\mu C_{p}}{k_{f}}, B i=\frac{h_{f}}{k_{f}}\left(\sqrt{\frac{v}{a}}\right) \\
& R d=\frac{4 \sigma^{\circ} T_{\infty}^{3}}{\beta_{R} k_{f}}, Q=\frac{Q^{\prime} v}{a}, E c=\frac{\rho u_{w}^{2}}{C_{p}\left(T_{w}-T_{\infty}\right)}, S c=\frac{v}{D_{B}}, k r=\frac{k_{r}^{\prime}}{a}, \alpha=\alpha^{\prime} \mu \sqrt{\frac{a}{v}}, f_{w}=-(a v)^{1 / 2} v_{w}
\end{aligned}
$$

are the Grashof number, Magnetic parameter, buoyancy ratio, micropolar parameter,

Micropolar parameters, dimensionless spin gradient viscosity parameter, Electric parameter, Prandtl number, convective heat transfer constant, radiation parameter, heat source parameter, Eckert parameter, Schmidt number, chemical reaction parameter, slip parameter, suction/injection velocity.

The local Nusselt number and Sherwood number on the wall are defined by

$$
N u_{x}=\frac{x q_{w}}{k_{f}\left(T_{w}-T_{\infty}\right)}, S h_{x}=\frac{x m_{w}}{D_{B}\left(C_{w}-C_{\infty}\right)}
$$

Where $\mathrm{q}_{\mathrm{w}}$ (heat flux), $\mathrm{m}_{\mathrm{w}}$ (mass flux) are given by

$$
q_{w}=-k_{f}\left(\frac{\partial T}{\partial y}\right)_{y=0,} m_{w}=-D_{B}\left(\frac{\partial C}{\partial y}\right)_{y=0,}
$$

Which in the non-dimensional form reduces

$$
N u_{x} \operatorname{Re}_{x}^{1 / 2}=-\theta^{\prime}(0), \quad S h_{x} \operatorname{Re}_{x}^{1 / 2}=-\phi^{\prime}(0)
$$

Where the local Reynolds number is given by $\operatorname{Re}_{x}=\frac{u_{w} x}{v}$

\section{METHOD OF SOLUTION:}

The Galerkin finite element method has been implemented to obtain numerical solutions of coupled non-linear equations (9) to (12) of third-order in $f$ and second order in $h, \theta, \phi$ under boundary conditions (13\&14). This technique is extremely efficient and allows robust solutions of complex coupled, nonlinear multiple degree differential equation systems. The assumed equations can be solved by any of the numerical technique viz. Gaussian elimination, LU Decomposition method etc.

VARIATIONAL FORMULATION :

The variation form associated with the equations (15)-(19) over a typical two nodded line at element $\left(\eta_{e}, \eta_{e+1}\right)$ is given by

$$
\begin{gathered}
\int_{\eta_{e}}^{\eta_{e+1}} w_{1}\left(f^{\prime}-j\right) d \eta=0 \\
\int_{\eta_{e}}^{\eta_{e+1}} w_{2}\left((1+\Delta) j^{\prime \prime}+0.5\left(f j^{\prime}-j^{2}\right)+G(\theta+N \phi)+\Delta h^{\prime}+M^{2} E-M^{2} j\right) d \eta=0 \\
\int_{\eta_{e}}^{\eta_{e+1}} w_{3}\left(\lambda h^{\prime \prime}-\frac{\lambda}{G_{1}}\left(2 h+j^{\prime}\right)-0.5\left(j f-f h^{\prime}\right)\right) d \eta=0 \\
\int_{\eta_{e}}^{\eta_{e+1}} w_{4}\left(\left(1+\frac{4 R d}{3}\right) \theta^{\prime \prime}+0.5 P_{r} f \theta^{\prime}-Q \operatorname{Pr} \theta+M^{2} \operatorname{Pr} E c\left(j^{2}+E^{2}-2 E j\right)\right) d \eta=0 \\
\int_{\eta_{e}}^{\eta_{e+1}} w_{5}\left(\phi^{\prime \prime}+0.5 S c\left(\phi^{\prime} f\right)+S c S o \theta^{\prime \prime}-\gamma S c \phi\right) d \eta=0
\end{gathered}
$$


Where $\mathrm{w}_{1}, \mathrm{w}_{2}, \mathrm{w}_{3}, \mathrm{w}_{4}, \mathrm{w}_{5}$ are arbitrary test functions and may be regarded as the variations in $\mathrm{f}, \mathrm{h}, \omega, \theta$ and $\phi$ respectively.

\section{FINITE ELEMENT FORMULATION}

The finite element method may be obtained from (15)-(19) by substituting finite element approximations of the form

$$
f=\sum_{k=1}^{3} f_{k} \psi_{k}, h=\sum_{k=1}^{3} h_{k} \psi_{k}, \theta=\sum_{k=1}^{3} \theta_{k} \psi_{k}, \phi=\sum_{k=1}^{3} \phi_{k} \psi_{k}
$$

We take $\mathrm{w}_{1}=\mathrm{w}_{2}=\mathrm{w}_{3}=\mathrm{w}_{4}=\mathrm{w}_{5}=\psi_{i}^{j}(i, j=1,2,3)$

Expressing $f^{k}, h^{k}, \theta^{k}, \phi^{k}$ in terms of local nodal values (20) and integrating the integral we obtain we obtain local stiffness matrices. These matrices are assembled into a global matrices using inter element continuity, equilibrium conditions and boundary conditions. The global matrices are solved by iteration procedure. This procedure is stopped if the absolute value of the difference between any two consecutive values is less than $10^{-6}$.

\section{DISCUSSION OF THE NUMERICAL RESULTS:}

In this analysis we analyse the effect of partial slip, thermal radiation, dissipation, on convective heat and mass transfer flow of a viscous, electrically conduction fluid past a stretching sheet in the presence of heat source with convective boundary condition .Results are acquired by allotting the values of dimensionless parameters as $\mathrm{G}=2, \mathrm{M}=0.5, \mathrm{~N}=1.0$, $\mathrm{Sc}=0.24, \gamma=0.5, \mathrm{~A}=0.2, \mathrm{f}_{\mathrm{w}}=0.2, \mathrm{~B}_{\mathrm{i}}=0.1, \mathrm{E}_{1}=0.5, \mathrm{Q}=2, \mathrm{G}_{1}=0.2, \lambda=0.2, \Delta=1, \mathrm{~S}=0.1, \mathrm{Rd}=0.5$, $\mathrm{Ec}=0.01, \operatorname{Pr}=0.71$, we have taken these values as common for the complete study of results, unless otherwise shown in respective plots and tables. The results are presented graphically in figures. 1a to $8 \mathrm{~d}$ for different parametric variations. Comparison of the present results with previous works are performed and excellent agreements have been obtained. The non-linear coupled differential equations are solved by Galerkin finite analysis with three nodded line segments. In the absence of convective heat and mass transfer $(\mathrm{N}=0, \mathrm{G}=0)$ the results are compared with Ramzan et al(18).

Figs.1a-1d represent the velocity, microrotation velocity, temperature and concentration with buoyancy $\operatorname{ratio}(\mathrm{N})$. It is found that when the molecular buoyancy force dominates over the thermal buoyancy force the fluid velocity and micropolar velocity increase in the flow region, when the buoyancy forces are in the same direction and for the forces acting in opposite directions, they depreciate in the flow region. The temperature and concentration reduces with increase in $\mathrm{N}>0$ and enhance with $\mathrm{N}<0$ in the entire flow region.(figs.1c,1d).

Figs.2a-2d shows the influence of dissipation on the velocity, micropolar velocity, temperature and concentration. It is pointed out that the presence of Eckert number, the velocity and micropolar velocity are increases (figs. $2 \mathrm{a} \& 2 \mathrm{~b}$ ). This is due to the fact that the thermal energy is reserved in the fluid on account of friction heating. Hence the velocity and micropolar velocity rises in the entire boundary layer. However, the the temperature increases and mass concentration reduces marginally with increase in Ec (fig.2c,2d).

Figs.3a-3d exhibit the variation of $f^{\prime}, \omega, \theta$ and $\mathrm{C}$ with convective heat transfer constant $\mathrm{Bi}$. We find from the profiles that an increase in $\mathrm{Bi}$ reduce the velocity, and enhances the micropolar velocity, temperature and the concentration in the flow region.

Figs. $4 \mathrm{a}-4 \mathrm{~d}$ show the variation of $f^{\prime}, \omega, \theta$ and $\mathrm{C}$ with microrotation parameter $(\Delta)$. From the profiles we find that the velocity and micropolar velocity reduce with increase in $\Delta$, while the temperature, concentration increase in the flow region.. 
From figs.5a-5d we notice a reduction in the velocity, temperature and concentration with increase in dimensionless vortex viscosity $\operatorname{parameter}(\lambda)$. The micropolar velocity reduces with increase in $\lambda$ in the region $(0,1)$ and increase with $\lambda$ in the remaining region

From figs.6a-6d represent the variation of $f^{\prime}, \omega, \theta$ and $\mathrm{C}$ with $\mathrm{S}$. It can be seen from the profiles that an increase in $S$ enhances the velocity and reduce the temperature and concentration in the flow region and also microrotation reduces in the viscosity of the boundary and enhances for way from it.

Figs.7a-7d depicts the influence of radiative heat flux (Rd) on the velocity, microrotation velocity, temperature and concentration. It is observed that higher the radiative heat flux larger the fluid velocity and micropolar throughout the boundary layer. This may be attributed to the fact that an increase in thermal radiation parameter increases thickness of the boundary layer. The presence of the thermal radiation is very significant on the variation of temperature. It is seen that the temperature rapidly increases in the presence of thermal radiation parameter throughout the thermal boundary layer. The thickness of the boundary layer also increases in the presence of Rd. The effect of Rd on concentration is to diminish it in the solute boundary layer.

From figs.8a-8d we find that the velocity and concentration reduces, the micropolar velocity and temperature enhances in the degenerating chemical reaction case. In the generating chemical reaction case, the velocity, micropolar velocity, concentration enhances , while the temperature produces in the flow region.

The rate of heat transfer (Nusselt number) at the wall $\eta=0$ is evaluated for different parametric variations. Higher the dissipation/radiative heat flux /convective heat transfer constant Bi lesser the Nusselt number at the wall. The Nusselt number reduces with increase in micropolar parameters $\Delta$ and $\lambda$.The rate of heat transfer enhances with increase in the buoyancy ratio $\mathrm{N}>0$ and reduces with $\mathrm{N}<0$.

The rate of mass transfer (Sherwood number) at the wall $\eta=0$ is evaluated with variations in different parameters. Higher the dissipation /slip parameter A / convective heat transfer constant Bi larger the rate of mass transfer at the wall. An increase in the micropolar parameter $\lambda$ smaller the rate of mass transfer at the wall. When the molecular buoyancy force dominates over the thermal buoyancy force Sh enhances when the buoyancy forces are in the same direction and for the forces acting in opposite directions, it reduces on the wall.

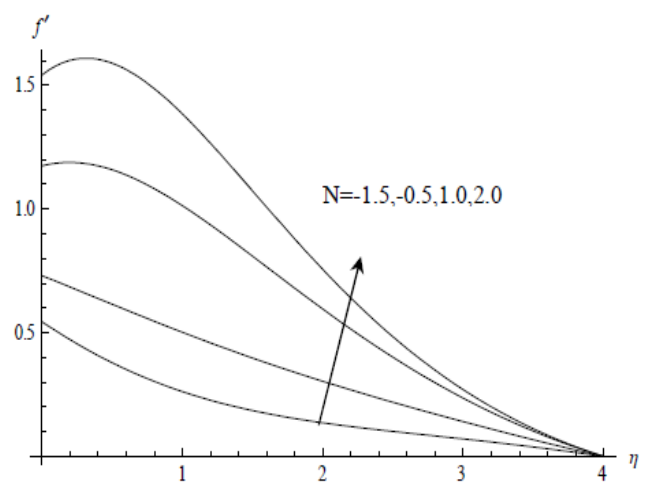

Fig.1a :Variation of $f^{\prime}$ with $\mathrm{N}$.

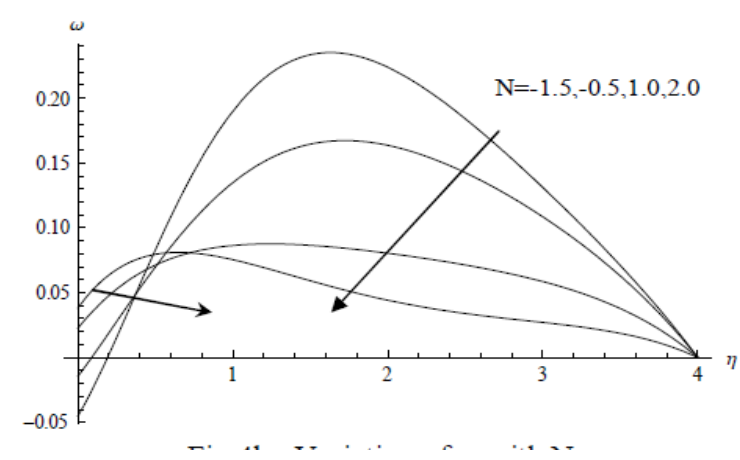

Fig.1b: Variation of $\omega$ with $\mathrm{N}$ 


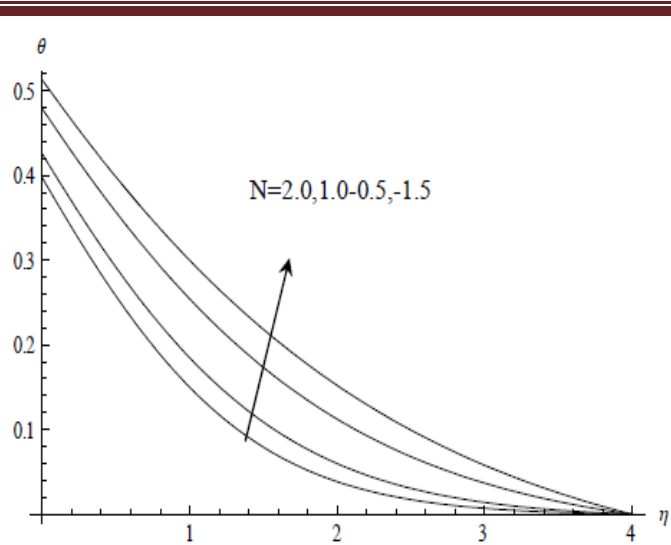

Fig.1c: Variation of $\theta$ with $\mathrm{N}$

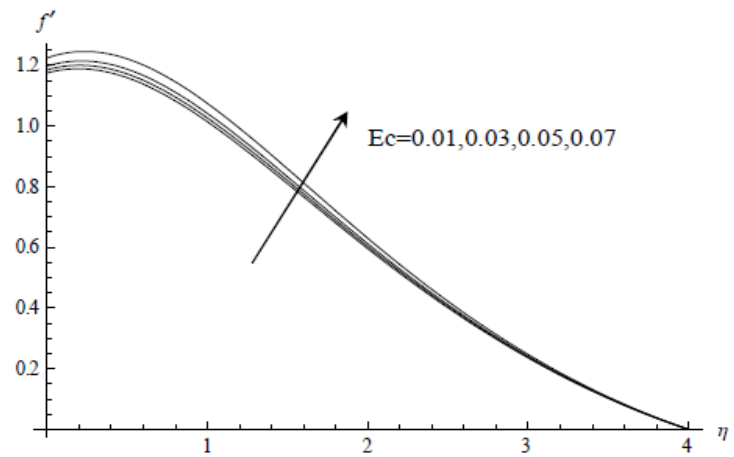

Fig.2a Variation of $f^{\prime}$ with Ec.

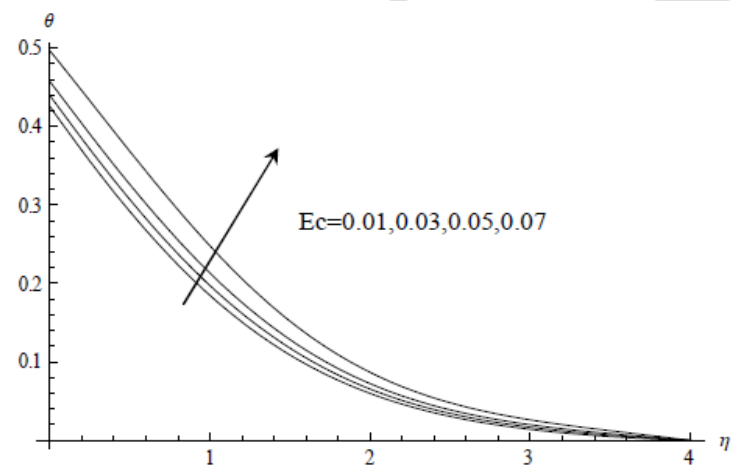

Fig.2c: Variation of $\theta$ with Ec

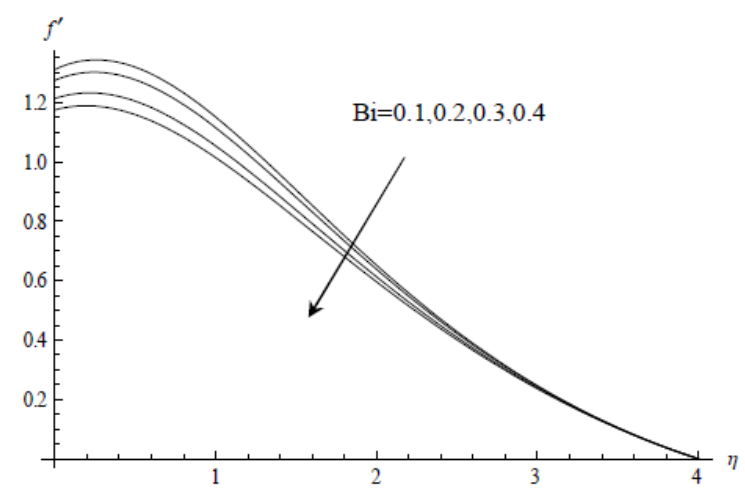

Fig.3a: Variation of $f^{\prime}$ with Bi.

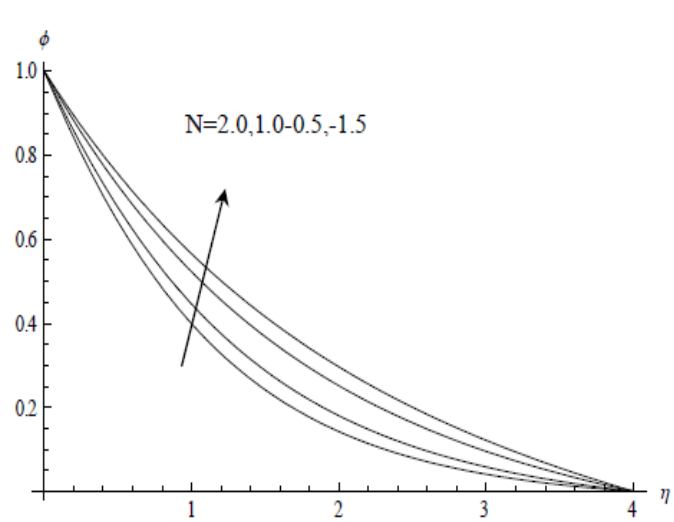

Fig.1d : Variation of $\phi$ with $\mathrm{N}$

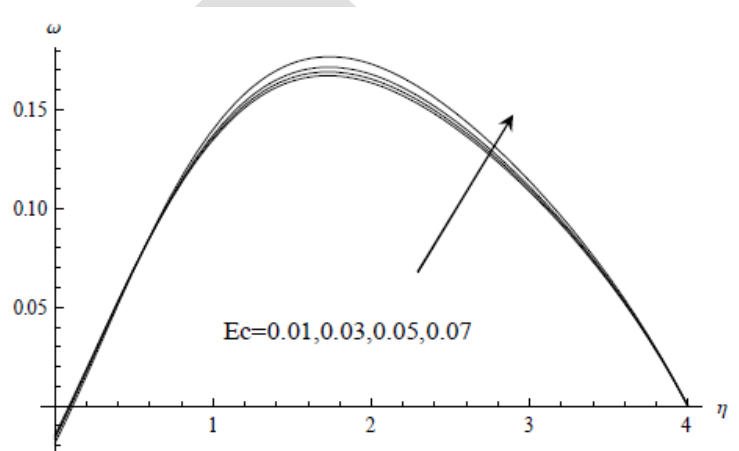

Fig.2b: Variation of $\omega$ with Ec.

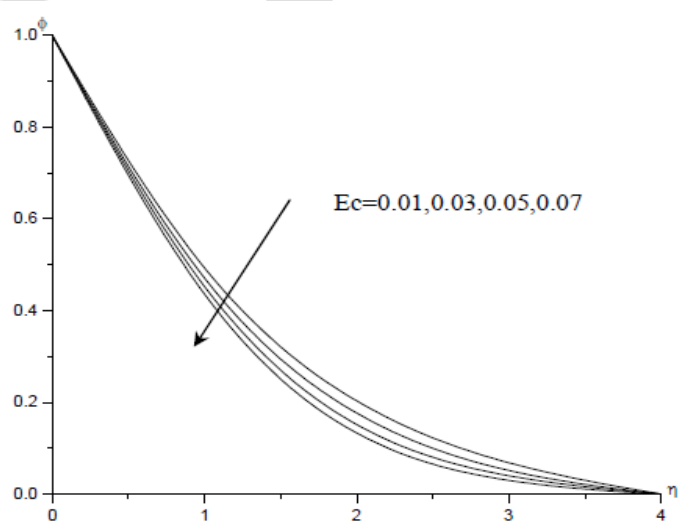

Fig.2d: Variation of $\phi$ with Ec

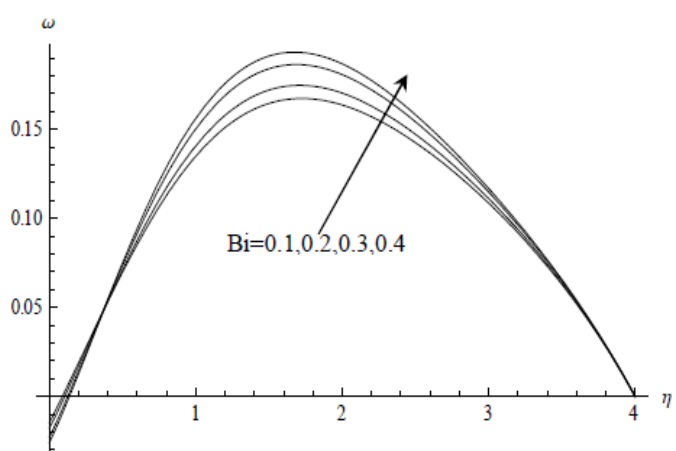

Fig.3b: Variation of $\omega$ with $\mathrm{Bi}$ 


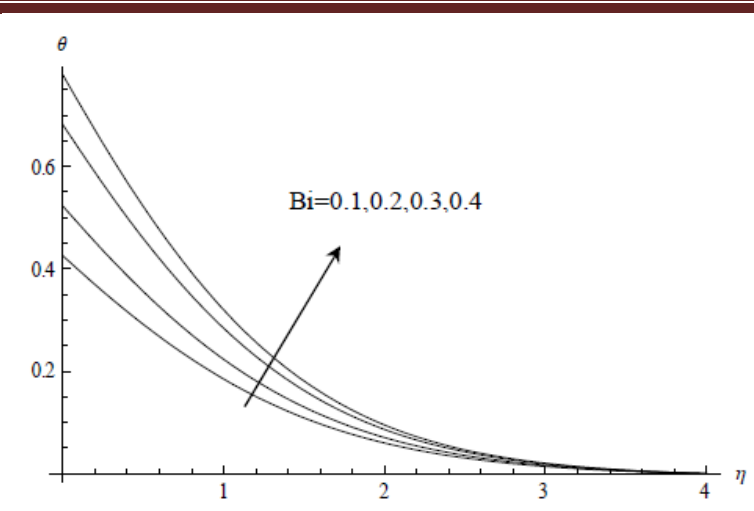

Fig.3c: Variation of $\theta$ with $\mathrm{Bi}$

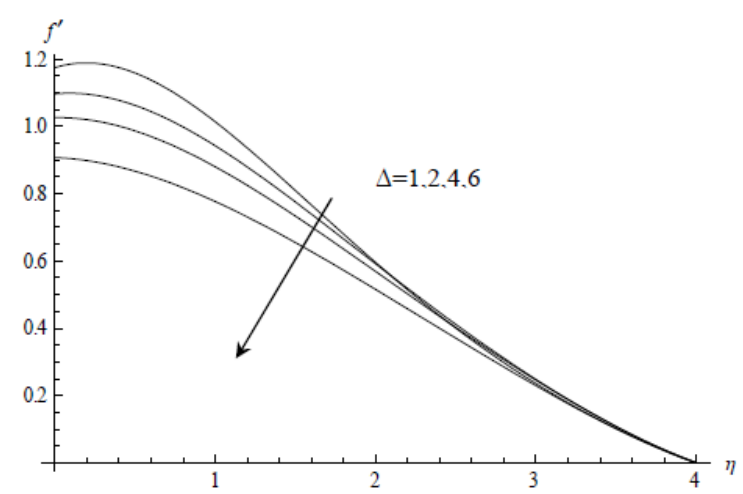

Fig.4a: Variation of $f^{\prime}$ with $\Delta$.

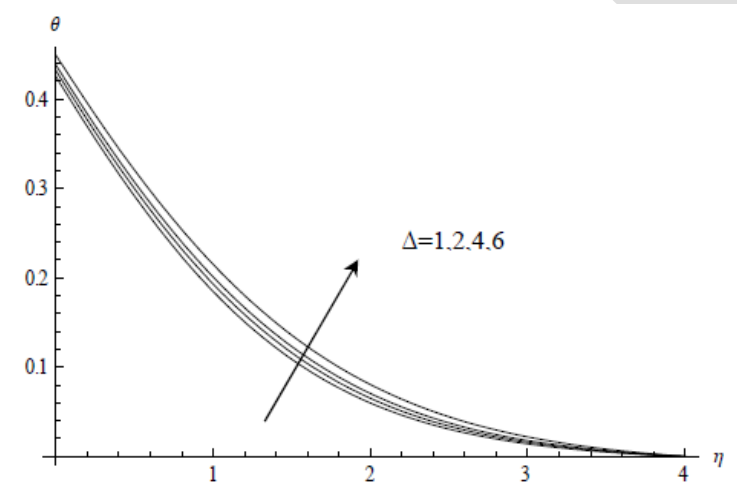

Fig.4c : Variation of $\theta$ with $\Delta$

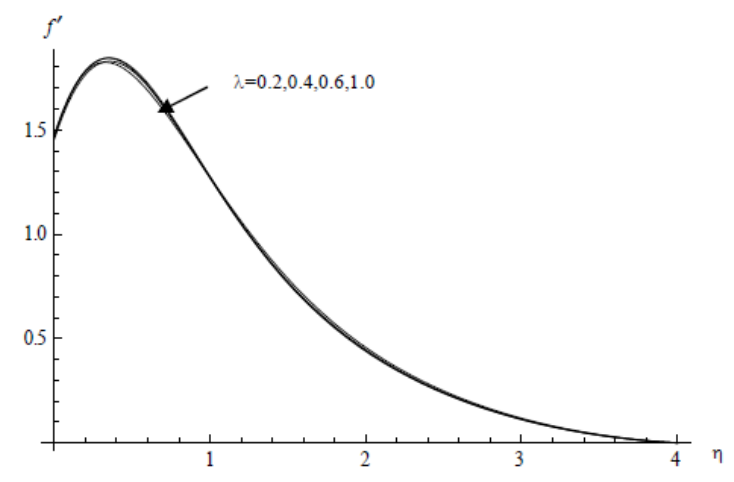

Fig.5a : Variation of $f^{\prime}$ with $\lambda$.

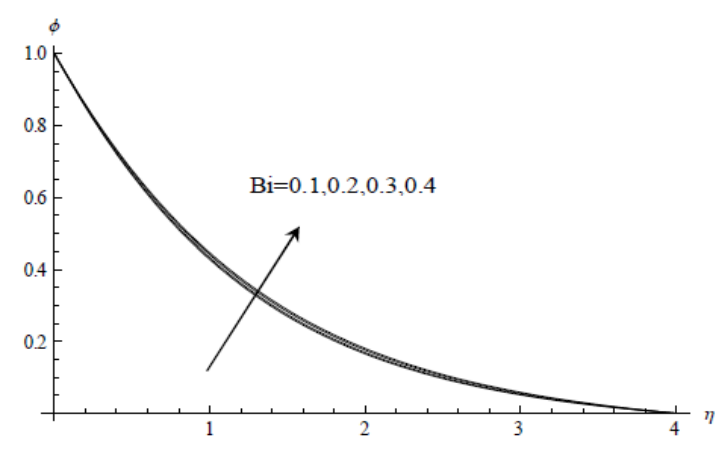

Fig.3d: Variation of $\phi$ with Bi

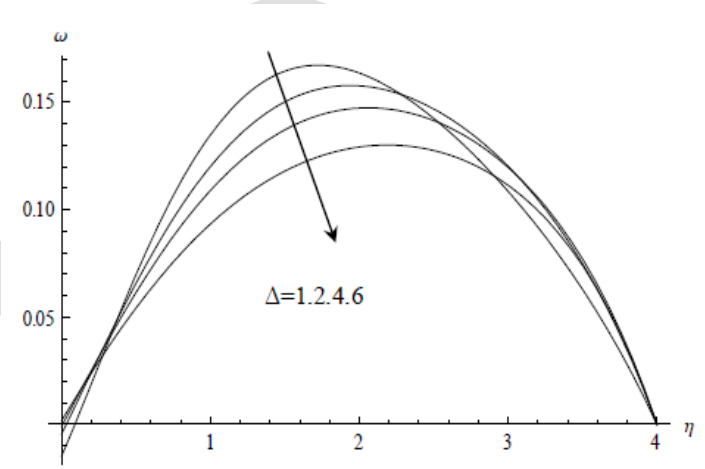

Fig.4b: Variation of $\omega$ with $\Delta$

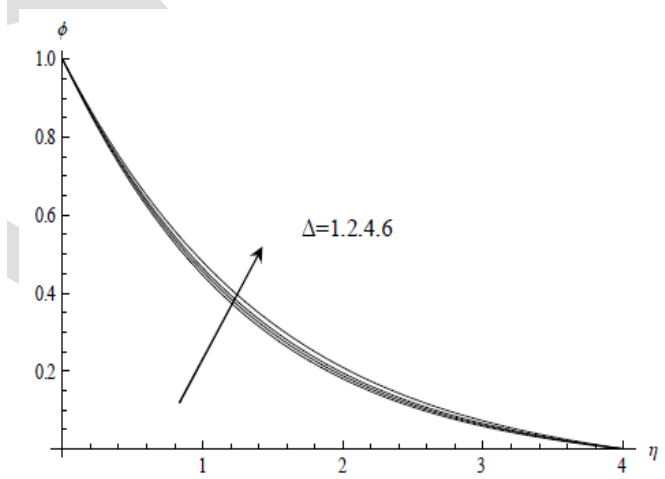

Fig.4d: Variation of $\phi$ with $\Delta$

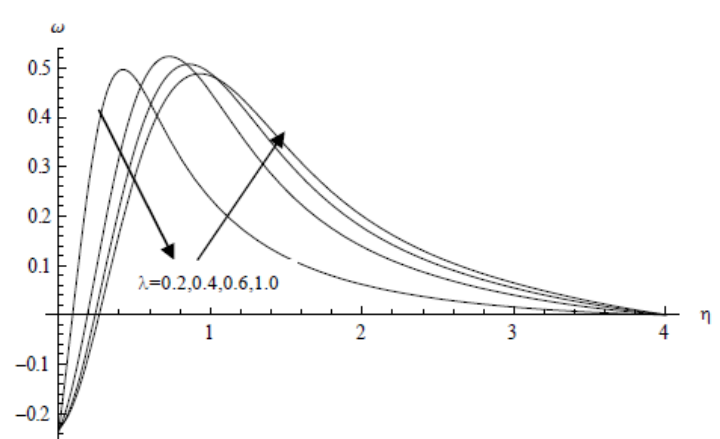

Fig. $5 b$ : Variation of $\omega$ with $\lambda$ 


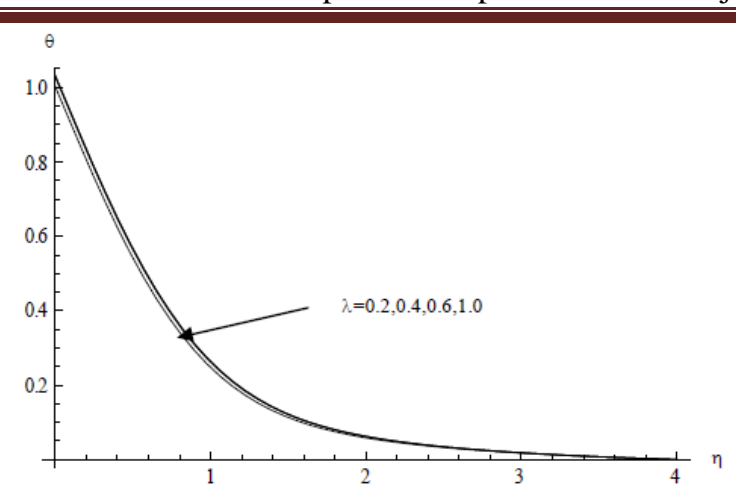

Fig.5c Variation of $\theta$ with $\lambda$.

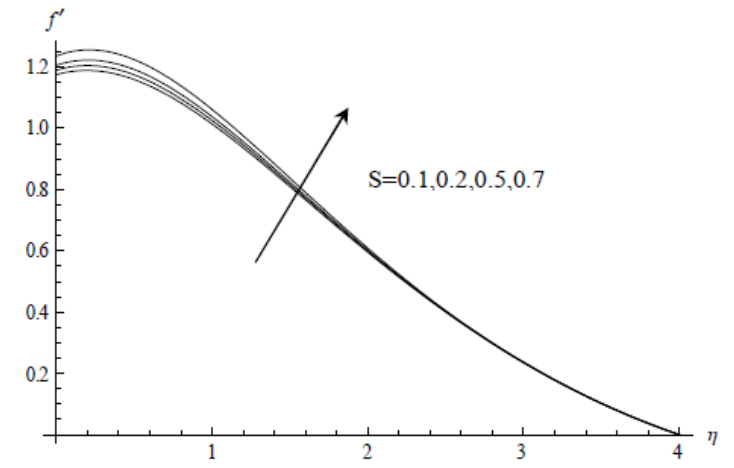

Fig.6a :Variation of $f^{\prime}$ with $\mathrm{S}$

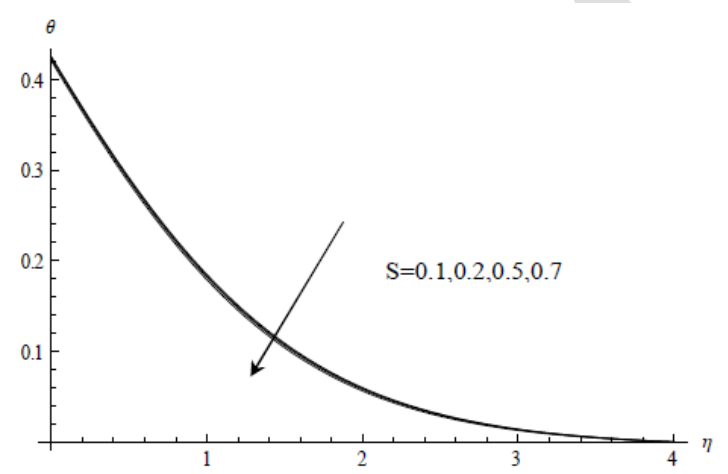

Fig.6c Variation of $\theta$ with S.

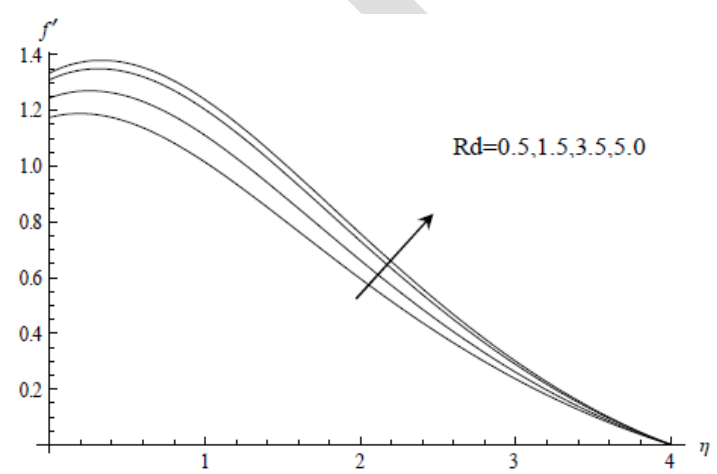

Fig.7a :Variation of $f^{\prime}$ with Rd

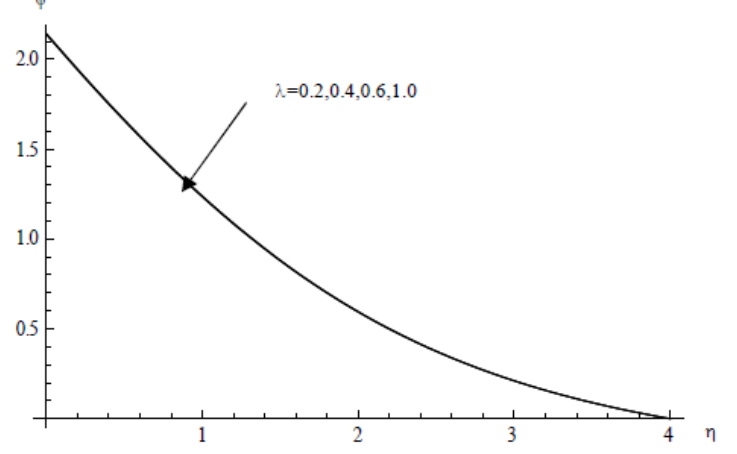

Fig.5d :Variation of $\phi$ with $\lambda$.

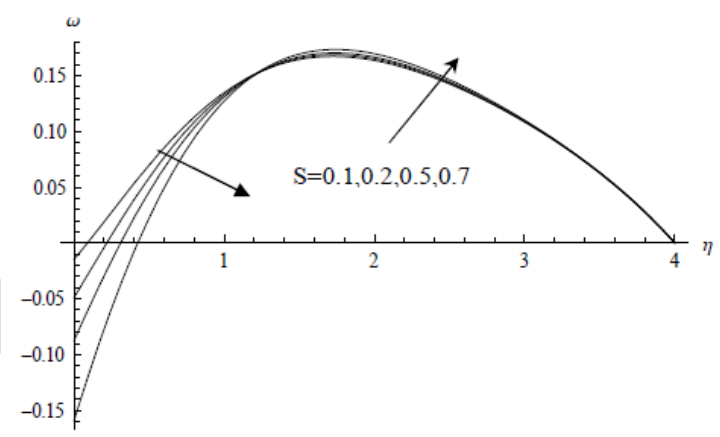

Fig.6b : Variation of $\omega$ with S

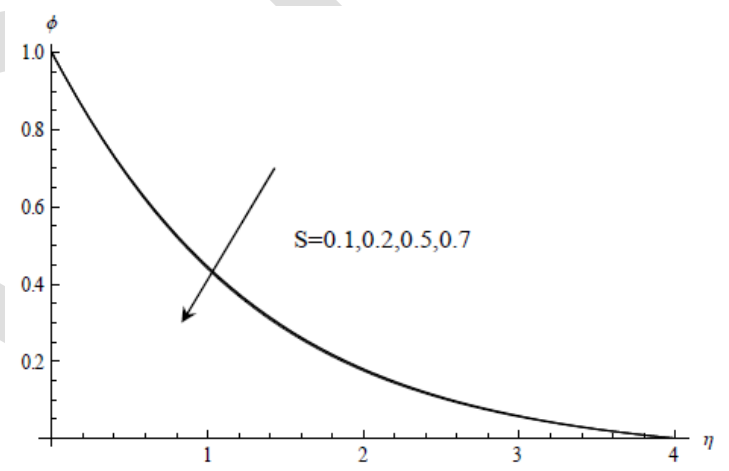

Fig.6d :Variation of $\phi$ with S.

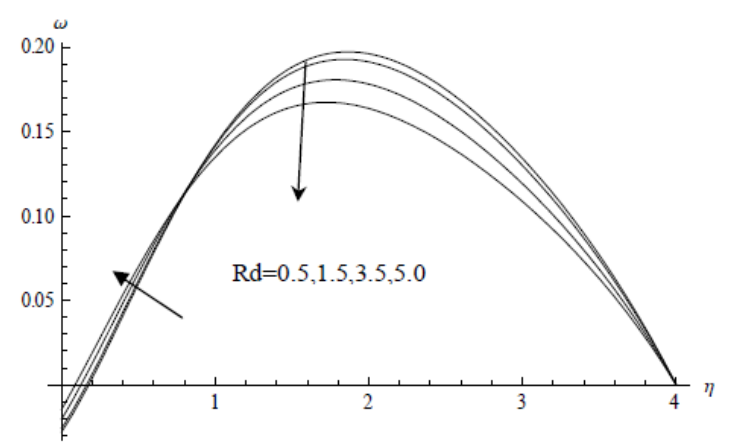

Fig. 7b : Variation of $\omega$ with $\mathrm{Rd}$ 


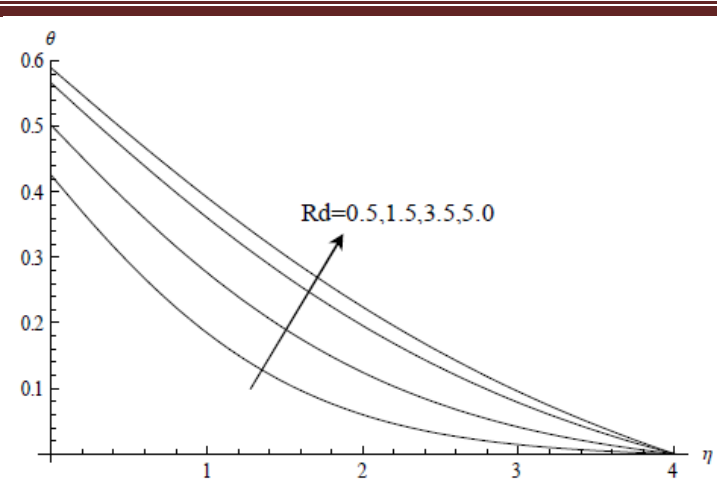

Fig.7c Variation of $\theta$ with $\mathrm{Rd}$

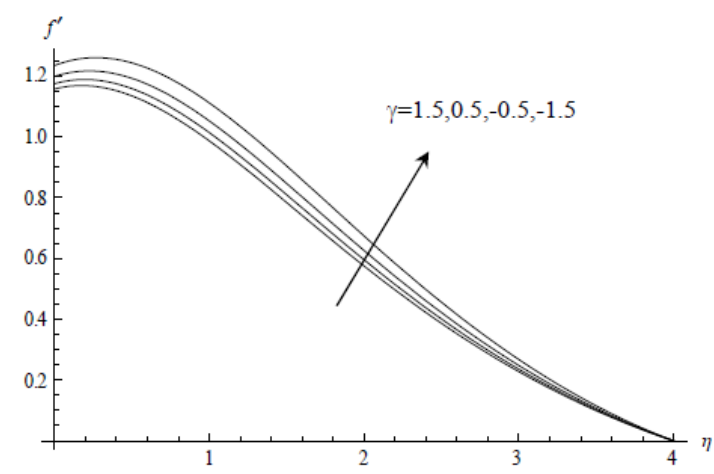

Fig.8a :Variation of $f^{\prime}$ with $\gamma$

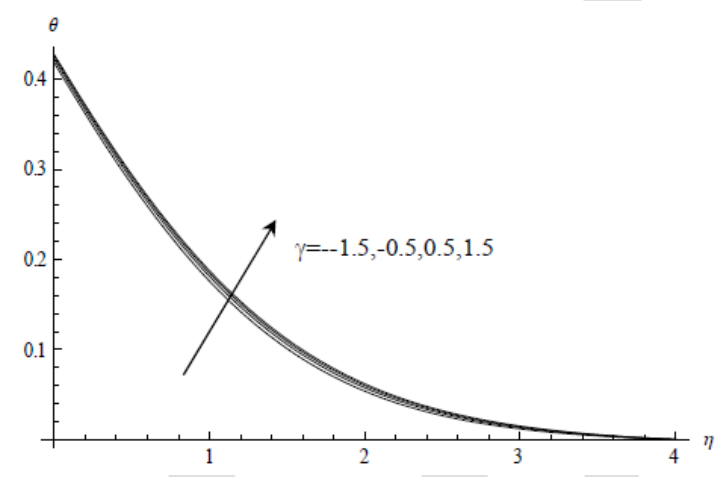

F ig.8c Variation of $\theta$ with $\gamma$

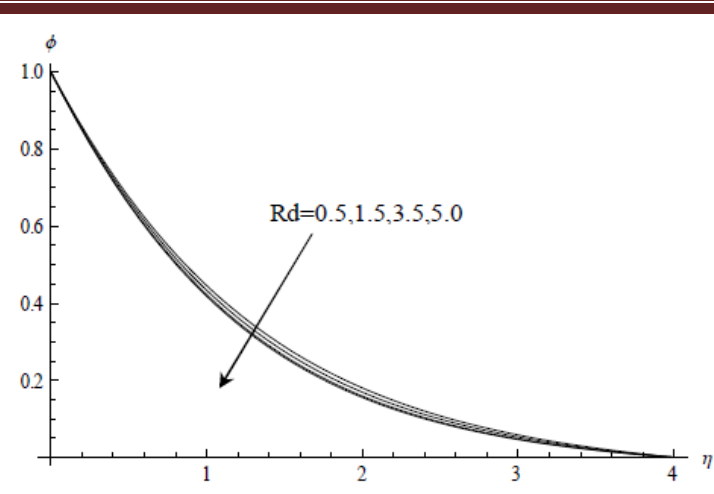

Fig.7d :Variation of $\phi$ with $\mathrm{Rd}$.

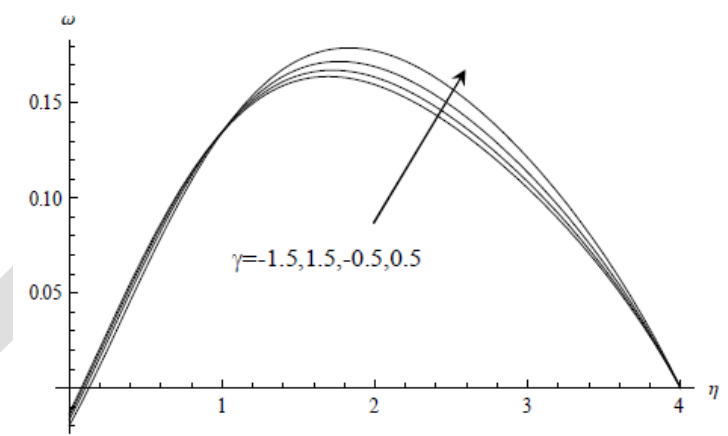

Fig.8b : Variation of $\omega$ with $\gamma$

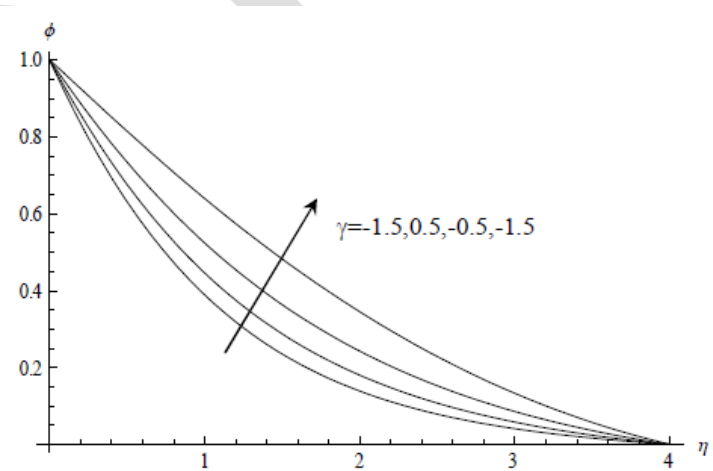

Fig.8d : Variation of $\phi$ with $\gamma$

Table 1: Nusselt Number and Sherwood Number at $\eta=0$

\begin{tabular}{|l|c|c|l|c|c|c|c|c|}
\hline $\mathbf{N}$ & $\mathbf{B i}$ & $\boldsymbol{\gamma}$ & $\boldsymbol{\lambda}$ & $\boldsymbol{\Delta}$ & $\mathbf{R d}$ & $\mathbf{E c}$ & $\mathbf{N u}$ & $\mathbf{S h}$ \\
\hline 1.0 & 0.1 & 0.5 & 0.2 & 1 & 0.5 & 0.01 & 2.35148 & 0.755958 \\
\hline 2.0 & 0.1 & 0.5 & 0.2 & 1 & 0.5 & 0.01 & 2.51783 & 0.847112 \\
\hline-0.5 & 0.1 & 0.5 & 0.2 & 1 & 0.5 & 0.01 & 2.08875 & 0.622624 \\
\hline-1.5 & 0.1 & 0.5 & 0.2 & 1 & 0.5 & 0.01 & 1.95077 & 0.554713 \\
\hline 1.0 & 0.3 & 0.5 & 0.2 & 1 & 0.5 & 0.01 & 1.46803 & 0.781488 \\
\hline 1.0 & 0.5 & 0.5 & 0.2 & 1 & 0.5 & 0.01 & 1.28449 & 0.790545 \\
\hline 1.0 & 0.7 & 0.5 & 0.2 & 1 & 0.5 & 0.01 & 1.20456 & 0.795224 \\
\hline 1.0 & 0.1 & 1.5 & 0.2 & 1 & 0.5 & 0.01 & 2.34026 & 0.894663 \\
\hline 1.0 & 0.1 & -0.5 & 0.2 & 1 & 0.5 & 0.01 & 2.367515 & 0.588693 \\
\hline 1.0 & 0.1 & -1.5 & 0.2 & 1 & 0.5 & 0.01 & 2.39044 & 0.3722467 \\
\hline 1.0 & 0.1 & 0.5 & 0.4 & 1 & 0.5 & 0.01 & 2.14567 & 0.807659 \\
\hline
\end{tabular}




\begin{tabular}{|c|c|c|c|c|c|c|c|c|}
\hline 1.0 & 0.1 & 0.5 & 0.6 & 1 & 0.5 & 0.01 & 2.03456 & 0.865437 \\
\hline 1.0 & 0.1 & 0.5 & 0.8 & 1 & 0.5 & 0.01 & 1.99876 & 0.93456 \\
\hline 1.0 & 0.1 & 0.5 & 0.2 & 2 & 0.5 & 0.01 & 2.33243 & 0.745768 \\
\hline 1.0 & 0.1 & 0.5 & 0.2 & 4 & 0.5 & 0.01 & 2.31811 & 0.738176 \\
\hline 1.0 & 0.1 & 0.5 & 0.2 & 6 & 0.5 & 0.01 & 2.28195 & 0.719205 \\
\hline 1.0 & 0.1 & 0.5 & 0.2 & 1 & 1.5 & 0.01 & 1.99361 & 0.777299 \\
\hline 1.0 & 0.1 & 0.5 & 0.2 & 1 & 3.5 & 0.01 & 1.76697 & 0.797179 \\
\hline 1.0 & 0.1 & 0.5 & 0.2 & 1 & 5.0 & 0.01 & 1.69826 & 0.804561 \\
\hline 1.0 & 0.1 & 0.5 & 0.2 & 1 & 0.5 & 0.03 & 2.32543 & 0.756935 \\
\hline 1.0 & 0.1 & 0.5 & 0.2 & 1 & 0.5 & 0.05 & 2.29181 & 0.758223 \\
\hline 1.0 & 0.1 & 0.5 & 0.2 & 1 & 0.5 & 0.07 & 2.25063 & 0.759836 \\
\hline
\end{tabular}

\section{CONCLUSIONS:}

1) when the molecular buoyancy force dominates over the thermal buoyancy force, the fluid velocity and micropolar velocity increase in the flow region, when the buoyancy forces are in the same direction and for the forces acting in opposite directions, they depreciate in the flow region. The temperature and concentration reduces with increase in $\mathrm{N}>0$ and enhance with $\mathrm{N}<0$ in the entire flow region. The Nusselt number, sherwood number enhances, the couple stress reduces with increase in $\mathrm{N}>0$ and for $\mathrm{N}<0$, the couple stress enhances, Nusselt number, Sherwood number reduces on the wall.

2) An increase in $\mathrm{Bi}$ enhances the velocity, micropolar velocity, temperature and reduces the concentration in the flow region. An increase in $\mathrm{Bi}$ enhances the couple stress, and reduces the rate of heat and mass transfer on the wall.

3) The velocity reduces with increase micropolar parameters $\Delta, \lambda$ and enhances with G1. An increase in $\Delta, \lambda, \mathrm{G} 1$ reduces the micro-rotation. The temperature enhances with increase in $\Delta, \mathrm{G} 1$ and reduces with. $\lambda$. The concentration enhances with $\Delta$ and reduces with $\mathrm{G} 1, \lambda$. An increase in $\Delta, \mathrm{G} 1$ and $\lambda$ reduces the couple stress. The Nusselt number reduces with $\Delta, \mathrm{G} 1$ and enhances with $\lambda$. The Sherwood number enhances with G1, $\lambda$ and reduces on the wall with $\Delta$.

4) Higher the thermal radiation larger the velocity, mocro-rotation, temperature and smaller the concentration in the flow region. The Sherwood number enhances while the Nusselt number, couple stress reduces with increase in radiation parameter $\mathrm{Rd}$.

5) The velocity $f^{\prime}$, the couple stress, the temperature enhances, the concentration reduces in the degenerating chemical reaction case while in the generating chemical reaction case the velocity, temperature and concentration while the micropolar velocity $(\omega)$, reduces in both degenerating and generating chemical reaction cases in the entire flow region. The Nuselt number, Sherwood number enhances, the couple stress reduces on the wall in the degenerating chemical reaction case while in the generating chemical reaction case, the couple stress enhances, the rate of heat and mass transfer reduces on the wall.

\section{REFERENCES:}

1. Alam MS, Rahman MM: Dufour and Soret effects on mixed convection flow past a vertical porous plate with variable suction., Nonlinear Anal Model Contr.,V.11,pp.312(2006).

2. Aman,F Ishak,A and Pop: Mixed convection boundary layer flow near stagnation poni on vertical surface with slip.,Appl.Maths and Mechs-Engnl.Ed,V.32,No.12,pp.15991606(2011).

3. Anderson,H.I:Slip flow past a stretching surface.,Acta.Mech,V.158,No.1,pp.121125(2002). 
4. Bourich M, Hasanaoui M, Amahmind A: Soret convection in a shallow porous cavity submitted to uniform fluxes of heat and mass .,Int Common Heat Mass transfer,V.31,pp.773-782(2004).

5. Chaudary R.C and Jain P, "An exact solution to the un-steady free- convection boundarylayer flow past an impulsively started vertical surface with Newtonian heating", J.Eng. Phys.Thermophys, 2007, 80, 954-960.

6. Dulal Pal and Sewli Chatterjee:Mixed convection magnetohydrodynamic heat and mass transfer past a stretching surface in a micropolar fluid-saturated porous medium under the influence of ohmic heating ,Soret and Dufour effects.,Commun Nonlinear Sci Numer Simulat,V.16,pp.1329-1346(2011).

7. El-Arbawy HAM.Soret and Dufour effects o natural convection flow past a vertical surface in a porous medium with variable surface temperature.,J Math Stat,V.5(3),pp.190198(2009).

8. Erigen ,AC:Theory of micropolar fluids.,J.Math Mech,V.16,pp.1-18(1968).

9. Hyot JW,Fabula AG:The effect of additives on fluid friction,US Naval Ordinace Test Station Report,USA.

10. Ibrahim Yakubu Seini and Daniel Oluwole Makinde :Boundary layer flow near stagnation -point on a vertical surface with slip in the presence of transverse magnetic fields.,Int.Jour.Numerical Methods for Heat and Fluid flow,V.24,No.3,pp.643-655(2014).

11. Lukaszewicz,G:Micropolar fluids-Theory and Apllications,Birkhauser,Basel(1999).

12. Psoteinicu A:Influence of chemical reaction on heat and mass transfer by natural convection from vertical surfaces in porous media considering Soret and Dufour effects.,Heat Mass transfer,V.43,pp.595-602(2007).

13. Reddy GM,Reddy BN:Soret and Dufour effects on steady MHD free stagnation point flow past a semi-infinite moving vertical plate in a porous medium with viscous dissipation.,Int J Appl Math Mech.V.6(1),pp.1-12(2964).

14. Rajesh V and Chamkha Ali J. "Unsteady convective flow past an exponentially accelerated infinite vertical porous plate with Newtonian heating and viscous dissipation", Int. Journal of Numerical Methods for Heat \& Fluid Flow,V. 24(5), pp.11091123(2014).

15. Srinivasarya,D and Upendra,M: Free convection in Mhd micropolar fluid with Soret and Dufour effects..Int.J.of Appl.Math and Mech.,V.((5),pp.92-112(2013).

16. Sulochana, G and Ramakrishna,G.N:Effect of non-linear density variation on non-Darcy convective heat and mass transfer with Newtonian cooling., International Journal Chemical and Process Engineering Research, V.40, pp. 1-12(2016).

17. Tsai R,Huang JS:Heat Mass transfer for Soret and Dufour effects on Hiemenz flow through a porous medium onto a stretching surface.,Int JHeat Mass transfer, V.52, pp.2399-2406(2009).

18. Ramzan,M,Farooq,M,Hayat,T and Jae Dong Chung:Radiative and Joule heating effects in the MHD flow of a micropolar fluid with partial slip and convective boundary condition.,Journal of Molecular liquids.,V.221,pp.394-400(2016). 